Production Planning \& Control

ISSN: 0953-7287 (Print) 1366-5871 (Online) Journal homepage: https://www.tandfonline.com/loi/tppc20

\title{
A survey of the assembly line balancing procedures
}

\section{Erdal Erel \& Subhash C. Sarin}

To cite this article: Erdal Erel \& Subhash C. Sarin (1998) A survey of the assembly line balancing procedures, Production Planning \& Control, 9:5, 414-434, DOI: $10.1080 / 095372898233902$

To link to this article: https://doi.org/10.1080/095372898233902

曲 Published online: 15 Nov 2010.

Submit your article to this journal ๘

Џll Article views: 1044

4 Citing articles: 233 View citing articles 


\title{
A survey of the assembly line balancing procedures
}

\author{
ERDAL EREL and SUBHASH C. SARIN*
}

Keywords assembly line, line balancing problem, heuristic procedure

\begin{abstract}
The assembly line balancing problem consists of assigning tasks to an ordered sequence of stations such that the precedence relations among the tasks are satisfied and some performance measure is optimized. Due to the complexity of the problem, heuristic procedures appear to be more promising than the optimum-seeking algorithms. For the single-model, deterministic version, there are numerous exact and heuristic algorithms developed, while for the other more complex but more realistic versions, the research published consists mainly of heuristic procedures. In this paper, the heuristic procedures are critically examined and summarized in sufficient detail to provide a state-of-the-art survey. An evaluation of the procedures and some further research topics have also been presented.
\end{abstract}

\section{Introduction}

The development of the first real example of an assembly line is credited to Henry Ford who developed such a line in 1913. But, for over 40 years since then, only trialand-error methods were used for balancing lines. Even by the early 1970s, as revealed in the survey of 95 companies made by Chase (1974), only 5\% of the companies were using published techniques to balance their lines. Since then the situation has not changed much; in a recent article, Milas (1990) states that companies design assembly lines manually either by 'gut feel' or historical precedent. This suggests that either currently available techniques are inadequate and/or inflexible to model the actual conditions of assembly lines, or the practitioners are unfamiliar with the published algorithms. This paper is written with a view to familiarize the reader

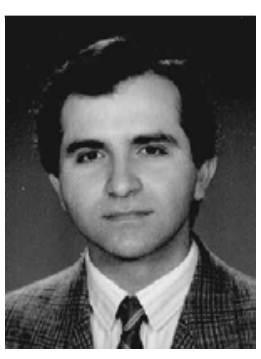

A uthors: E. Erel, Bilkent University, Faculty of Business Administration, 06533 Bilkent, Ankara, Turkey, and S. C. Sarin, Virginia Polytechnic Institute and State University, Department of Industrial and Systems Engineering, Blacksburg VA, 24061-0118, USA.

ERDAL EREL is an Associate Professor of the Faculty of Business Administration at Bilkent University, Ankara, Turkey. He received his $\mathrm{PhD}$ in Industrial Engineering and Operations Research from Virginia Polytechnic Institute and State University. His research interests are in the areas of manufacturing systems analysis, and production planning and control. He is a member of INFORMS.

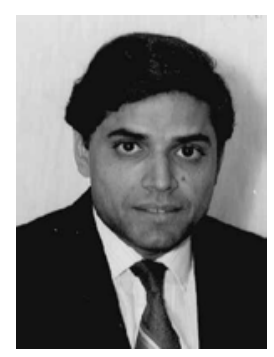

SubHash C. SARinis a Professor in the Department of Industrial and Systems Engineering at the Virginia Polytechnic Institute and State University. He received his PhD in Operations Research and Industrial Engineering from North Carolina State University, Raleigh, North Carolina. His areas of interest are production scheduling, applied mathematical programming and design, and mathematical analysis of manufacturing systems. He is a full member of INFORMS and a senior member of IIE.

\footnotetext{
* To whom correspondence should be addressed.
} 
with the state-of-the-art heuristic procedures for use in balancing assembly lines.

The first published analytical statement of the assembly line balancing problem (ALBP) was made by Salveson (1955) and followed by Jackson (1956), Bowman (1960), Supnik and Solinger (1960), White (1916), and $\mathrm{Hu}$ (1961). Since then, the topic of line balancing has been of great interest to academicians. Although extensive research has been done in the area, the problem has consistently defied the development of efficient algorithms for obtaining optimal solutions (Gutjahr and Nemhauser 1964). With the growth of knowledge on the subject, review articles are necessary to organize and summarize the findings for the researchers and practitioners. In fact, several articles (Kilbridge and Wester 1962a, Ingall 1965, Mastor 1970, Buxey et al. 1973, Johnson 1981, Yano and Bolat 1989) have reviewed the work published on the subject. The most recent review articles have been written by Baybars (1968b) who reviewed the exact algorithms for the basic (single-model and deterministic) problem, and by Ghosh and Gagnon (1989) who presented a comprehensive literature review of the subject. In this paper, we consider the heuristic procedures developed for the ALBP; the procedures considered are all critically examined and summarized in sufficient detail to provide a state-of-the-art survey.

Table 1. Symbols used in the paper.

\begin{tabular}{ll}
$B i$ & set of tasks that cannot start to be processed due to \\
& the incompletion of task $i$ \\
$C$ & cycle time \\
$D$ & demand rate \\
$E I C_{i}$ & expected incompletion cost of task $i$ \\
$f s_{i}$ & total number of starting events of task $i$ \\
$I C_{i}$ & incompletion cost of task $i$ \\
$K$ & number of stations on the line \\
$K_{\text {heu }}$ & number of stations found by a heuristic \\
$K_{\text {opt }}$ & optimal number of stations \\
$K_{j}$ & set of tasks assigned to station $j$ \\
$L$ & labour rate \\
$L_{\mathrm{a}}$ & overtime labour rate \\
$L C$ & labour cost \\
$N$ & number of tasks in the problem \\
$P_{c}$ & probability that a station time can exceed $C$ \\
$S_{i}$ & station time of station $j$ \\
$S_{\mathrm{max}}$ & maximum station time \\
$S B_{i}^{j}$ & correction factor for task $i$ and starting event $j$ \\
$t_{i}$ & performance time of task $i$ \\
$t_{\text {max }}$ & maximum task performance time \\
$T C$ & total cost \\
$Y$ & number of zeros in the precedence matrix \\
$\mu_{i}$ & mean of performance time of task $i$ \\
$\sigma_{\mathrm{i}}$ & standard deviation of performance time of task $i$ \\
$\beta_{i}$ & probability that task $i$ has started to be processed \\
& and not completed within $C$ \\
\hline
\end{tabular}

We will define and explain several terms and concepts related to the subject prior to the examination of the studies. Table 1 depicts the symbols used in the paper with their definitions. An assembly line can be considered as a production sequence where parts are assembled together to form an end product with the assembly operations being carried out at workstations situated along the line. A task is the smallest, indivisible and rational work element of the total work content in an assembly process. A station is a location on the line at which work is performed on the product either by adding parts or by completing the assembly operations. Stations can be classified as open and closed stations: it is undesirable, or impossible, for operators from adjacent stations to violate the boundaries of a closed station. Open station boundaries can be crossed, so there is flexibility in the times available for completing tasks, but it is required that no interference occurs between adjacent operators. Station time, $S_{j}$, is the actual amount of work, in time units, assigned to station $j$ on the line. Task performance times, $t_{i}$ is the duration of task $i$, and cycle time, $C$, is the amount of time a unit being worked on is available to an operator. The following bounds can be imposed on $C$ :

$$
\max _{i=1, \ldots, N} t_{i} \leq \max _{j=1, \ldots, K} S_{j} \leq C \leq 1 / D
$$

where $N$ is the number of tasks in the problem, $K$ is the number of stations on the line and $D$ is the demand rate. A precedence diagram is a graphical description of the ordering in which tasks must be performed in achieving the total assembly of the product. Prenting and Battaglin (1964) present a detailed description of precedence diagrams. A precedence matrix is an upper-triangular matrix which has an entry of one for the $i$ th row and $j$ th column if task $j$ follows task $i$ in the precedence diagram; otherwise, the entry is zero. The precedence structure of a problem can be characterized by the Flexibility-ratio ( $F$-ratio); it is a measure of the number of feasible sequences that could be generated from an $\mathrm{N}$ task problem, and can be expressed as follows:

$$
F \text {-ratio }=\frac{2 Y}{N(N-1)}
$$

where $Y$ is the number of zeros in the precedence matrix. A similar measure is the order strength expressed as follows:

$$
\text { Order strength }=\frac{2 \text { (Number of precedence relations) }}{N(N-1)}
$$

It measures the volume of distinct orderings that are permitted by the specified precedence relations. The ratio of average number of tasks assigned to stations is called the WEST-ratio, and is expressed as follows: 


$$
\text { WEST-ratio }=\frac{N}{K}
$$

Idle time is the difference between $C$ and the station time. It is conventional to take the sum of all station idle times as a measure of the efficiency of the line design; this sum is called the total idle time. A related measure of efficiency is balance delay, which is the ratio of the total idle time and the total time spent by the product in moving from the beginning to the end of the line (Kilbridge and Wester 1961a). It can be expressed as follows:

$$
\text { Balance delay }=\frac{100\left(K C-\sum_{i=1}^{N} t_{i}\right)}{K C}
$$

Rosenblatt and Carlson (1985) defined a similar term called the efficiency of the line, this is the complement of the balance delay. Another related measure of efficiency is the smoothness index, this can be expressed as follows:

$$
\text { Smoothness index }=\sqrt{\sum_{j=1}^{K}\left(S_{\max }-s_{j}\right)^{2}}
$$

The most commonly used line balancing objectives can be classified into two categories. The first category involves minimization of total idle time given a desired $C$. The second category involves minimization of $C$ for a fixed number of stations. The problem considered in the first category is sometimes called the Type I problem, and the one considered in the second category is called the Type II problem. Minimizing balance delay and maximizing the profit per unit of time (Rosenblatt and Carlson 1985) are some other objectives. Most of the procedures developed deal with finding the minimum number of workstations given a desired $C$. This objective, in fact, is equivalent to minimizing total idle time given a desired $C$ as shown below. The objective of minimizing total idle time can be expressed as follows:

$$
\operatorname{Min} Z=K C-\sum_{i=1}^{N} t_{i}
$$

The objective as stated above can be reduced to one of two alternative forms as follows:

(1) Min $Z=K$, given $C$; or

(2) Min $Z=C$, given $K$.

The reduction is due to the fact that $\sum_{i=1}^{N} t_{i}$ is a constant, and $K$ or $C$ are the only variables to be minimized. In most cases, $C$ is predetermined when management sets the production rate, or an upper bound is imposed by production planning requirements. Thus, the problem is reduced to finding the minimum number of stations subject to the constraints:

(1) All tasks have to be performed;

(2) The work content in any station cannot exceed $C$;

(3) Precedence relations are satisfied.

Although the problem is easy to formulate, the enumeration of the feasible task sequences to find the minimum number of stations requires an enormous effort. The problem has a finite but extremely large number of feasible solutions; this immense computational complexity and the problem's inherent integer restrictions result in enormous computational difficulties. Without the precedence constraints, there are $N$ ! different sequences of $N$ tasks. However, precedence and cycle time constraints reduce this figure drastically. As Ignall (1965) reports 'if there are $r$ precedence relations among $N$ tasks, then there are roughly $N ! / 2 r$ distinct sequences', which is still too large to handle.

ALBPs can be classified into four categories: SingleModel Deterministic (SMD), Single-Model Stochastic (SMS), Multi/mixed Model Deterministic (MMD) and Multi/mixed Model Stochastic (MMS). The SMD version of the problem applies to single-model assembly lines where the task performance times are known constants. This is the simplest form of the ALBP. The SMS category introduces the concept of task time variability. This version represents the manual assembly lines more realistically where task performance times are seldom constants. The variabilities of task times can be rather large relative to their means. This is especially true for the tasks which are complex and demand high levels of skill and concentration. The MMD version of the problem introduces the concept of producing more than one item on a single line. Multi-model lines are involved in the production of two or more similar types of items produced separately in batches, whereas mixed-model lines produce two or more similar items simultaneously. The MMS category is the most complex version of the problem to analyse. Figure 1 depicts the above classification with the solution procedures developed for each class. The labels below the boxes address the section numbers in which the procedures for the corresponding problem are discussed.

Another classification scheme is based on the way the items are moved through the line. In this scheme, there are basically two distinct types: non-mechanical and moving belt lines. Operators on non-mechanical lines are normally free of any mechanical pacing effect; the product remains stationary at each station. Moving belt lines are basically paced lines characterized by a conveyor belt; Kwo (1958) is one of the earliest researchers who has examined conveyors. 


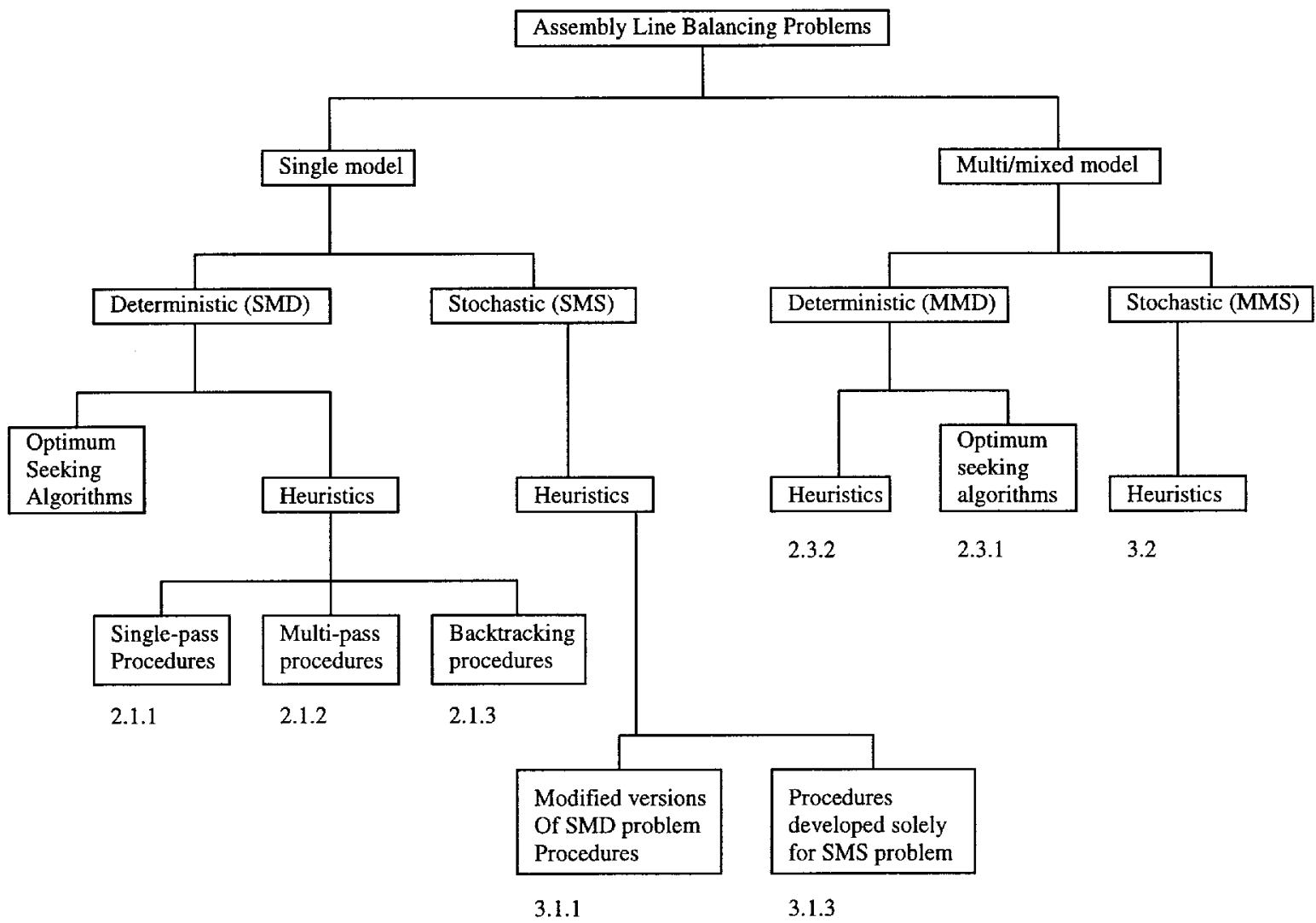

Figure 1. Classification of the assembly line balancing problem and the related solution procedures.

\subsection{Overview}

In this paper, we discuss the heuristic procedures developed for the paced SMD, SMS, MMD and MMS assembly line balancing problems in which no in-process inventory is allowed between stations. For the unpaced lines with/without in-process inventories, the reader is referred to the review paper of Dallery and Gershwin (1992). The reader is also referred to Smunt and Perkins (1985) who have examined lines with realistic environments via simulation.

In Section 2, the heuristic procedures developed for the deterministic problem and the related comparative studies are reviewed. In Section 2.1, the procedures developed for the SMD problem are reviewed, and in Section 2.2 the comparative studies to evaluate the SMD problem procedures are presented. In Section 2.3 , the procedures developed for the MMD problem are discussed. In Section 3, we discuss the stochastic ALBP and the related issues. Solution procedures suggested for the SMS and MMS problems are reviewed in Sections 3.1 and 3.2, respectively. Note that these sections are also addressed in figure 1 . In Section 4, we evaluate the procedures and give guidance on their use- fulness to practitioners and researchers, and point out some issues related with the ALBP that need to be studied further.

\section{Deterministic assembly line balancing problem}

The deterministic ALBP can be stated as follows: given a finite set of tasks, each having a fixed performance time, and a set of precedence relations which specify the permissible orderings of the tasks, the problem is to assign the tasks to an ordered sequence of stations such that the precedence relations are satisfied and some measure of performance is optimized.

The attempts to solve the deterministic ALBP can be classified into two groups. The first group consists of the algorithms that attempt to determine the optimal solution; the interested reader is referred to the survey article of Baybars (1986b). The second group consists of heuristic procedures that utilize principles or devices that contribute to the reduction of search in the problem-solving activity at a cost of not guaranteeing the optimal solution. In view of the computational complexity of the ALBPs in general, the heuristic procedures appear 
to be more promising than the optimum-seeking algorithms.

Numerous heuristic procedures have been reported with significantly different characteristics. These are reviewed by grouping the procedures of similar or close characteristics in Section 2.1. In Section 2.2, the comparative studies made to evaluate the procedures are reviewed. The procedures for the MMD problem are reviewed in Section 2.3.

\subsection{Heuristic procedures for the SMD problem}

The procedures are classified into three categories. The first category consists of single-pass decision rule procedures which implement a list processing prioritizing scheme for task assignment based on a single attribute of each task. The second category consists of procedures that produce multiple single-pass solutions and select the most attractive solution. The last category consists of procedures that attempt to improve a solution or a station assignment by some iterative backtracking methods. The procedures are reviewed according to the above classification, and presented in Sections 2.1.1, 2.1.2 and 2.1.3, respectively.

\subsubsection{Single-pass decision rule procedures}

Helgeson and Birnie (1961) proposed the well-known and popular 'Ranked Positional Weight Technique' (RPWT). In this technique, each task is given a weight equal to the sum of its task time and the task times of all other tasks that follow it on the precedence diagram. Tasks are listed in descending order of their weights and an attempt is made to assign the tasks to stations in that order. If a task takes longer than the time remaining in the station or would violate the precedence relations, then the next task is considered. If no further task can be assigned to the station, the next station is opened. To provide two line designs to choose from, the authors have proposed the idea of 'inverse positional weight' which is obtained by looking at the assembly operation from the end to the start of the line. Assignment to stations starts from the last station and proceeds forward from there. Although the technique does not even guarantee a near-optimal solution, it makes it possible to test many alternative balances by considering different $C$ values. In spite of the fact that the method is very popular in the literature (it appears in almost every comparative study and in several textbooks), Ignall (1965) reports that the method results in a solution far from the optimum for his example problem. Mastor (1970) also supports Ignall (1965), showing that the technique per- forms worse than almost all of the other techniques compared in his study.

Tonge $(1960,1961)$ developed a heuristic procedure for the problem consisting of three phases: (i) simplification of the initial problem by grouping adjacent tasks into compound tasks; (ii) solution of the more simple problems by assigning tasks to stations at the least complex level possible, breaking up the compound tasks into their elements only when necessary for a solution; and (iii) smoothing the resulting balance by transferring tasks among stations until the distribution of assigned time is as even as possible.

Kilbridge and Wester (1961b) proposed a technique developed primarily to balance lines without the aid of a computer. The main feature of the technique is to group tasks into columns in the precedence diagram where tasks are placed as far left as possible without violating the precedence relations. In such a diagram, tasks can be permuted among themselves in each column and some of the tasks can be moved laterally from their columns to positions to their right without violating the precedence relations. Then, two properties of the tasks in the diagram, permutability within columns and lateral transferability, are exploited in an attempt to achieve optimum balance. As Kilbridge and Wester (1961b) stated, the technique is not a mere mechanical procedure, since a fair amount of judgement and intuition must be used to derive a meaningful solution. It is a simple, powerful technique, especially for large $C$, when one station crosses several columns. On the other hand, for low $C$, where one column may require two or more stations, a fair amount of adjustment is necessary. Kilbridge and Wester (1962a) applied the technique to a problem taken from industry in which fixed facilities and positional restrictions exist. They (1961b) also examined the relation of balance delay with various problem parameters, e.g. the range of task time, $C$, degree of precedence relation flexibility. They report that balance delay is very sensitive to the right selection of $C$.

Agrawal (1985) developed a procedure which utilizes a decision rule called 'largest set rule' for allotting the work to stations. The procedure computes the cumulative time for each task which is the time for performing the task and all the tasks preceding it. Then, the largest cumulative time which is less than $C$ is selected and the associated tasks are assigned to the worker. The procedure is repeated on the truncated precedence diagram until all the tasks are assigned. After the work is allotted to workers, the designer should decide on the sequence in which these workers should be positioned on the line. Although the procedure is computer efficient, there is no apparent guarantee of yielding a good solution.

Baybars (1986a) developed a procedure that consists of five phases. The first four phases reduce the size of the 
problem by utilizing various properties of the problem and the last phase is a single-pass heuristic procedure applied on the reduced problem. The procedure starts with the last tasks in the precedence diagram and proceeds backward. The tasks with the most unassigned immediate predecessors among the tasks with no unassigned followers are assigned first. Detailed computational results on the 70-task problem of Tonge (1961) are presented, as well as the results of other problems reported in the literature. The results indicate that the procedure finds the optimal solution in most of the cases with minimal computation time.

\subsection{2. $M$ ultiple single-pass solution procedures}

Tonge (1965) proposed a procedure which assigns tasks to stations by randomly selecting a heuristic procedure for choosing the next task to add to the current station. Based on three example problems and by considering different $C$ values, it is reported that the random selection of heuristics for choosing the next task does as well as or better than, either using an individual heuristic procedure alone, or randomly choosing the tasks without an intervening choice of heuristic procedures.

Arcus (1966) developed a technique called 'Computer Method of Sequencing Operations for Assembly Lines' (COMSOAL), in which the main idea is the random generation of a feasible sequence. The technique assigns the same probability of selection to the tasks with no unassigned predecessors and fits the remaining station time. Judging on the basis of the yield of good balances, the author has explored methods of biasing the tasks available for selection. Among the nine methods developed, the one which is a combination of the others gives the best results. Results are reported for a 1000-task problem with a known optimal 200 stations with zero idle time, and the maximum possible number of tasks available for assignment being 69 , using about half of the capacity of an IBM 7094 computer, a sequence requiring 203 stations (1.48\% idle time) was achieved in $2 \mathrm{~min}$.

Buxey (1978) improved COMSOAL further with parallelling of stations leading to possible reductions in total idle time. He also applied the same approach to the RPWT of Helgeson and Birnie (1961). Each station that is duplicated is assumed to have an effective cycle time of $C$ times the station multiple. Thus, a range of times becomes available and there is more likelihood of a better fit. Multiple stations also enable the production rate to be greater than the limitation imposed by $t_{\max }$.

Nkasu and Leung (1995) developed a procedure similar to COMSOAL in the sense that the best design is selected among the several generated via simulation. Performance measures of minimizing $K, C$, balance delay, and a combination of these are considered. The procedure allows the task times and $C$ to be sampled from various probability distributions. Neither an experimentation nor comparison of the procedure with the others in the literature is given; thus, it is impossible to comment on the performance of the procedure.

Schofield (1979) developed a procedure called 'Nottingham University Line Sequencing Program' (NULISP) that is also similar to COMSOAL. NULISP can solve both Type I and II problems, handle various zoning constraints and task times larger than $C$. The details of the feasible sequence generation are not reported due to copyright reasons; however, it is stated that a weighted random selection procedure is utilized to generate solutions. The major advantage of NULISP is the feature of considering various factors, e.g. grouping of tasks for a variety of reasons, separation of one group of tasks from others for reasons of skill differences, safety considerations, and fixing of tasks at certain stations to account for fixed facilities on the line.

Pinto et al. (1978) presented a heuristic network procedure based on the shortest-route formulation of Gutjahr and Nemhauser (1964) in which the nodes represent a collection or subset of tasks that can be performed in some order without prior completion of any task not in the subset. The directed arc $(i j)$ is defined if $U_{i}$ is a subset of $U_{j}$ and $t\left(U_{j}\right)-t\left(U_{i}\right) \leq C$, where $U_{i}$ and $t\left(U_{i}\right)$ represent the set of tasks at node $i$ and the sum of their task times, respectively. Pinto et al. (1978) have utilized other heuristic procedures, e.g. RPWT, largest task time, smallest task time and random assignment, to generate the nodes. The set of nodes generated is combined to form a composite network. The procedure is reported to result in a balance delay of $96.8 \%$ for a 50 -task problem.

Akagi et al. (1983) proposed a method which allows the assignment of more than one worker to a station. Tasks are assigned to stations according to a couple of rules reported in the literature. The procedure is repeated for a different number of workers at each station. In the second phase of this two-phase technique, tasks are assigned to workers within each station.

\subsubsection{Backtracking procedures}

Hoffman (1963) developed an enumeration method which generates all feasible station assignments that do not exceed $C$ and selects the best arrangement from among these by use of a triangular precedence matrix. The procedure selects as the first station the feasible subset of tasks that leaves the least idle time, then selects from the remaining tasks the subset that leaves the least idle time in the second station, etc. The method is coded in FORTRAN that can solve problems with up to 99 tasks. Although the method may be computationally very expensive, Gehrlein and Patterson (1978) demonstrate 
that the method, suitably modified, could be used to solve problems of moderate sizes.

Moodie and Young (1965) developed a two-phase procedure. In the first phase, a preliminary balance is obtained by selecting the task with no unassigned predecessors and fitting the remaining station time in the order of largest performance time. In the second phase, tasks are shifted between stations in an attempt to reduce idle time and distribute the idle time equally to all stations. Similar to this procedure, Sarker and Shanthikumar (1983) developed another procedure that enables the balancing of lines some involving task times greater than $C$.

Nevins (1972) developed a general purpose heuristic program called the 'best bud search' that does not attempt to minimize the number of stations directly; instead, an upper bound on the number of stations is imposed and the problem is solved for that many stations. If the attempt is successful, the number of stations is decremented by one, and another attempt is made until it is either impossible or computationally impractical to get a smaller number of stations. Nevins (1972) tested the problems solved by Tonge (1961) and obtained as good or better results.

Dar-El (1973) developed a method called MALB for the Type II problem. The method starts with the minimum theoretical $C$ and proceeds with the generation of a feasible sequence of tasks which are grouped into station assignments. The method aims at grouping all the tasks into the required number of stations. If a feasible sequence cannot be extended, the method applies a backtracking procedure which either partitions the tasks correctly or results in an increase of one time unit of $C$. The method is further improved by imposing rules which limit the backtracking iterations. This method is shown to dominate COMSOAL and 10-SPP (a method selecting the best of 10 solutions, each obtained by using a different ranking system, e.g. as with RPWT) in the problems tested. Dar-El (1975) compared MALB with COMSOAL and single-pass methods, e.g. RPWT, and obtained consistently superior results over the others.

Dar-El and Rubinovitch (1979) developed another method which generates alternative solutions of equal quality by employing exhaustive enumeration to generate all or some subset of the solutions. However, the computational requirements of the exhaustive enumeration grow exponentially with the number of subsets saved. In order to make it manageable, the sets are saved as sequences of bits and circular storage buffers are utilized. This method, called 'Multiple Solutions Technique' (MUST) dominates or gives equal quality results with MALB in every case.

Shtub and Dar-El (1990) utilized MALB for a multiobjective approach for both Type I and II problems. The objective functions consist of the traditional objective of minimizing the total idle time and minimizing the number of sub-assemblies handled at each station. The purpose of considering the second objective is to improve work methods and enrich employee jobs. Four models are developed: the first model has the objective of minimizing the weighted sum of the total number of subassemblies and $K$. The second model has the objective of minimizing the weighted sum of the total number of sub-assemblies and $C$. The third model minimizes $K$ subject to the additional constraint that all the stations do not handle more than a predetermined number of subassemblies. The last model minimizes $C$ with the same additional constraint of the third model. A heuristic procedure is developed based on MALB, and a set of zoning constraints is imposed on the problem so the tasks of specific sub-assemblies are restricted to a single station. An efficient frontier is obtained which maps the relationship between the total idle time and the corresponding solution having the minimum number of sub-assemblies handled by the line. It is noted that the choice of a solution from the efficient frontier is based on a trade-off between the effort to minimize the total idle time and the effort to minimize the total number of sub-assemblies.

Bennett and Byrd (1976) presented a two-stage 'trainable heuristic procedure'. In the first stage, the procedure is trained by accumulating experience on the effectiveness of several heuristic rules on small problems for which the optimum is known. In the second stage, the findings of the first stage are used to provide a near-optimal solution which is fed to an optimization procedure as a starting point. The authors have used several empirical rules and values with no apparent justification.

Hackman et al. (1989) developed a branch-and-bound procedure that incorporates several heuristic fathoming rules to reduce the size of the tree. The authors report that the procedure outperforms the other branch-andbound procedures in the literature on the 53 example problems solved. The procedure can also be adapted to solve the Type II problem.

Easton (1990) presented a dynamic programming (DP)-based approach with relaxation and fathoming that relies on a dynamic upper bound. It is well known that the DP formulation of the ALBP problems of realistic sizes requires excessive storage and computation time. However, utilizing an upper bound to prune some states of the formulation may reduce these requirements. Dynamic upper bounds determined by a heuristic procedure have been utilized for some promising states. The author showed that the requirements of the DP formulation with dynamic upper bounds are much less than those of the formulation with a static upper bound on several problems in the literature. 
Faaland et al. (1992) considered lines with resource alternatives for each station; task times at a station are considered to be the functions of the resource alternative selected for that station. The objective function includes the costs associated with the selected resource alternatives as well as the costs of establishing the stations. An optimal solution procedure based on the shortest-network formulation of Gutjahr and Nemhauser (1964) is presented that is capable of solving problems of limited sizes. The authors implement two heuristic procedures in the optimization procedure; the first considers a subset of possible dominant paths by limiting the number of nodes which are scanned at any stage, whereas the second fixes a task sequence prior to applying the optimization procedure. An experimental design is constructed to test the cost performance and the computational effectiveness of the heuristic procedures; 128 problems are solved with up to 30 tasks. Based on the experimentation, the authors conclude that the second heuristic procedure can lead to an attractive compromise between quality and computational effort.

Leu et al. (1994) used genetic algorithms (GA) to solve the SMD problem. Chromosomes represent the feasible ordering of the tasks with each gene on the chromosome corresponding to a single task. Six single-pass heuristics taken from the literature are utilized to generate the parent population. Efficiency of the balance is utilized as the fitness function, although the authors report that other objectives can be easily considered simultaneously. Roulette-wheel selection is used to select the chromosomes for recombination and mutation in which the feasibility of the offsprings are guaranteed. An experiment on 40 randomly generated problems (with 50 or 100 tasks) and GA parameters each having three levels of settings resulting in $3240 \mathrm{GA}$ solutions has been conducted. The best GA solution outperforms the best of the six singlepass heuristic solutions in 14 of the 40 problems. The authors recommend to utilize as many heuristics as possible to generate the parent population and to try several different levels of mutation rate. The authors claim that the results above have been achieved with 500 iterations; if it had been with more iterations, more than 14 problems would have outperformed. A comparison with the other effective heuristic procedures in the literature would be useful to assess the effectiveness of the GA approach.

Anderson and Ferris (1994) presented a GA application on the SMD problem to demonstrate the potential of GAs to solve combinatorial optimization problems. The gene in the $i$ th place of the chromosome corresponds to the station to which the $i$ th task is to be assigned. Chromosomes are selected for cross-over and mutation with the 'Stochastic Universal Sampling'; cross-over occurs at a single point with a prespecified probability and then the offsprings undergo mutation. Utilizing a randomly generated initial population outperforms other initial populations generated by heuristic procedures. The authors report the results of a detailed experimentation conducted on problems with 50 tasks. The main aim of the study is to show the effects of various GA variables on the performance of the algorithm.

Scholl and Voß (1996) presented a bidirectional prioritizing scheme for task assignment by building a forward and backward ranking. The backward ranking is analogous to the 'inverse positional weight' of Helgeson and Birnie (1961). At each iteration, the task with the highest priority (obtained either in forward and backward ranking) is assigned to the station. When all the tasks are assigned, the partial forward and backward stations are combined. The author also used tabu search to solve the Type II problem. A neighbourhood is defined as a shift of a task from one station to another or exchanging two tasks between different stations. The tabu search procedure is iteratively applied to solve the Type I problem. The search procedure improves the initial solutions obtained by the bidirectional heuristic procedures on the large set of problems solved.

2.2. Comparative studies to evaluate the SMD problem heuristic procedures

The first article which reviewed and compared the SMD problem procedures was published by Kilbridge and Wester (1962b). They defined numerous terms related to the problem, and reviewed LP formulations of the problem made by Salveson (1955) and Bowman (1960), a DP formulation made by Jackson (1956), and heuristic procedures developed by Helgeson and Birnie (1961), Tonge (1960, 1961), and Kilbridge and Wester (1961b).

Later, Ignall (1965) reviewed the procedures reported in the survey article of Kilbridge and Wester (1962b), COMSOAL, the DP formulation of Held et al. (1963), and the heuristic procedure of Moodie and Young (1965). He pointed out the complications arising from mixed-model lines and variable performances times.

Mastor (1970) compared the procedures of Arcus (1966), Held et al. (1963), Hoffmann (1963), Kilbridge and Wester (1961b), and Helgeson and Birnie (1961) by solving a series of problems. The problems are generated by varying the number of tasks, number of stations (line length) and order strengths. The performance measures are taken as the output rate (equivalently, cycle time) and the cost of computation. It was found that there are consistent differences among the results of the procedures and line length, order strength, and the number of tasks have significant effect on the results. The best 
effectiveness results are consistently achieved by the procedure of Held et al. (1963), followed by COMSOAL.

Dar-El (1975) compared three different classes of balancing procedures for the Type II problem; the first class, called 10-SP, selects the best of 10 single-pass solutions, the second class is COMSOAL, and the last class is MALB. COMSOAL is used by allowing 75 sequence generations for each cycle time, and if a solution is not obtained, the cycle time is raised by one time unit and another 75 sequences are generated. The figure of 75 is chosen considering the computation costs. The set of problems is generated by considering different numbers of tasks, $F$-ratios and WEST-ratios. The measures used for solution efficiency are the balance delay and computation times. MALB gave consistently superior results than the other procedures, and COMSOAL was generally shown to dominate the 10-SP. On the other hand, COMSOAL required the maximum computation time.

Johnson (1981) compared six balancing procedures; three of them are DP procedures, two are branch-andbound procedures, and the last one is the shortest-route procedure developed by Gutjahr and Nemhauser (1964). Two sets of problems are generated with 20 tasks and 40 tasks in each problem by varying the cycle times and the order strengths. In the first set with 20-task problems, the branch-and-bound procedures and the DP procedure of Jackson (1956) perform better than the others. In the second set with 40 -task problems, none of the procedures is able to solve the problems with six or more tasks per station within $20 \mathrm{~s}$ of computation time on an IBM $360 / 91$.

Talbot et al. (1986) compared four classes of procedures for the Type I problem. The first class consists of single-pass decision rule procedures which implement a list processing prioritizing scheme for task assignment based on a single attribute of each task. The second class consists of procedures which utilize combinations of some single-pass decision rules, or procedures which produce multiple single-pass solutions and then select the best solution among them. The third class consists of some backtracking procedures which attempt to improve station assignments previously obtained. The last class consists of optimum-seeking algorithms with a time limit set to restrict the time allowed for each solution. Four sets of problems are generated for comparison purposes: the first set consists of sixty 50-task and sixty 100 -task problems with different order strengths, cycle times and task times. The second set consists of the problems reported in the literature ranging in size from 7 to 111 tasks. The third and fourth sets are called 'difficult problem sets' that consist of 50- and 100-task problems with few precedence relations and a wide range of task times. The authors concluded that using a backtracking procedure and then improving the solution with an opti- mum-seeking procedure would be the best policy if computer speed and storage are limiting factors.

Hoffmann (1990) developed a set of problems to provide a suitable vehicle for comparing existing algorithms or testing new ones. In order to evaluate the difficulty level of the problems, an effective exact algorithm was developed based on the heuristic procedure of Hoffmann (1963). Problems of various difficulty levels are generated by varying the problem size, $C$, precedence relations and task times. It is also noted that neither of these factors by themselves can predict difficulty. The difficulty level is determined by the interaction of all these factors, the solution procedure and its implementation.

Scholl and Voß (1996) compared the forward, backward and bidirectional prioritizing rules for task assignment for the Type I and II problems on a large set of problems, of which some are reported in the literature. For the Type I problem, bidirectional procedures outperform both forward and backward prioritizing procedures. For the Type II problem, the authors test various search methods that solve Type I problem instances to determine the minimum $C$. The lower bound search method which starts with $C=\max \left\{\sum_{i} / K ; t_{\max }\right\}$ and increments by 1 if no feasible solution is found outperforms the other search methods.

\subsection{Procedures for the $M M D$ problem}

Kilbridge and Wester (1962b) were among the first researchers to mention the MMD version of the problem in which two or more similar models are produced in batches or simultaneously. Balancing of mixed-model lines is a problem encountered in large sections of industry, notably the automotive companies. In this version of the problem, a model sequencing decision has also to be made in addition to the task allocation problem (e.g. see Dar-El and Cother 1975, Dar-El and Cucuy 1977, DarEl and Nadivi 1981).

Dar-El (1978) presented a classification of the MMD problem and the main design features, e.g. the conveyance system, the product's link to the conveying system, the types of stations, and the launching discipline.

\subsubsection{Optimum seeking algorithms}

Due to the large computational and storage requirements of the MMD version of the problem, few researchers have attempted to obtain the optimal solution. Roberts and Villa (1970) constructed an integer programming model; the objective is taken as the minimization of the total idle time. Due to the excessive number of constraints and variables, they have concluded that the 
formulation is of more theoretical than practical interest. For example, a two-model problem involving a total of 16 tasks and 16 precedence constraints requires 126 variables and 60 constraints. The authors also modified the network model of Gutjahr and Nemhauser (1964) developed for the SMD problem to handle the MMD problem. The computational and storage requirements of the procedure increase considerably for the MMD problem.

Gokcen and Erel (1997) presented a binary integer programming model that utilizes a combined precedence diagram for the different models in the problem. The model is remarkably superior to the model of Roberts and Villa (1970), and optimal solutions of problems with up to 40 tasks in the combined diagram have been reported.

\subsubsection{Heuristic procedures}

Arcus (1966) considered mixed-model lines as one of the complex forms of the problem and implemented COMSOAL, developed for the SMD problem, by using average task times obtained by multiplying the task times and the relative frequencies of the related models. He also considered the times necessary for changing tools and worker's position between units: the changing times are multiplied by the relative frequencies and added to the station times. However, no experimental results are reported.

Thomopoulos (1967) modified the procedure of Kilbridge and Wester (1961b), developed for the SMD problem, to balance the mixed-model problem. The modification consists of considering the total schedule for a whole shift and assigning tasks to stations on a shift basis rather than on a cycle time basis. He defined four kinds of inefficiencies: i.e. idleness, work deficiency, utility work and work congestion. Idleness occurs when the worker is kept idle waiting for work, work deficiency occurs when the worker is able to complete work before the next unit enters into the station, utility work and work congestion are related to the incompletion of the work due to time shortage. Thomopoulos (1967) has determined the sequence of the models by computing the penalty costs of inefficiencies resulting from sequencing the various models. The sequencing procedure is a heuristic one, but the author reported that the problems tested by Monte Carlo methods yield near-optimal solutions. The author has also reported the results of partitioning the schedule throughout the day, so all units of a model are not clustered in one segment of the sequence.

Thomopoulos (1970) later developed a procedure that assigns tasks to stations in a serial fashion. Precedence diagrams of the models are combined, and an equivalent single model system is obtained. For every station, the procedure searches a finite number of feasible combinations of tasks until an acceptable combination is obtained. An acceptable combination has a station time which falls into a predetermined interval. Then, an attempt to minimize fluctuations in station times of any given model among the stations is made by selecting the feasible combination which minimizes the deviation of the station time from the average station time. The procedure is also directly applicable to multi-model lines.

Chakravarty and Shtub (1986) developed a procedure which integrates labour cost with in-process inventory holding cost and machine setup cost for the unpaced MMD problem. Precedence diagrams of the models are combined to transform the system into a single-model system and then the tasks are ordered sequentially to transform the system into a serial system. Tasks are grouped to form the stations using either shortest-path approach or a single-pass heuristic procedure. The experimentation conducted reveals that the shortestpath approach yields designs with lower total costs. The experimentation also reveals that the shortest-path solutions have total costs which are on average within $3 \%$ of the lower bound found by relaxing the precedence relations and the integer multiple requirement of lot sizes.

Yano and Bolat (1989) developed a procedure motivated by the management of an automobile manufacturer. The objective is taken as the minimization of the total utility work; thus, it is optimal for a worker to complete as much of the task as possible before starting on the next task. The procedure proposed for the solution of the fomulation is a heuristic branch-and-bound procedure in which only the best node is retained at each level of the tree. Problems with parameters taken from the manufacturer are solved; the proposed procedure performs better than the currently used procedures by the manufacturer and another auto manufacturer.

Berger et al. (1992) presented a branch-and-bound algorithm with a truncated search for the MMD version in which the common tasks of the models are performed first. In other words, production processes of the models start diverging after the common tasks are performed. Several node generation schemes, of which one of them is based on the work of Hackman et al. (1989), are developed and tested. The authors also report the computational performance of these schemes.

\section{Stochastic assembly line balancing problem}

The stochastic ALBP can be stated as follows: given a finite set of tasks, each having a performance time distributed according to a probability distribution and a set of precedence relations which specify the permissible orderings of the tasks, the problem is to assign the tasks 
to an ordered sequence of stations such that the precedence relations are satisfied and some measure of performance is optimized.

When task performance times are random variables, a task may be incomplete due to two reasons: (1) the task is not completed within $C$; or (2) the task is a follower of another incomplete task on the precedence diagram. Note that decreasing $C$ or $K$ increases the number of incomplete tasks. Incompletion costs $(I C)$ depend on the way incompletions are handled. If incompletions are completed off the line, then the cost includes the labour cost of completing the task off the line, the cost of machinery used and the other related overheated costs. As stated by Kottas and Lau (1973), this approach closely approximates the cases often encountered in the assembly of automobiles and appliances. Other alternatives to handle incompletions are as follows: the entire line can be stopped for the time necessary to complete the incomplete task, products can be inspected and repaired at special stations strategically located along the line, or a generally-skilled team can serve as a mobile repair station to help where needed. As stated by Silverman and Carter (1986), for heavy, complex products, e.g. engines, the momentary stopping of the line is the economical alternative. On the other hand, if incompletions are scrapped, then the cost includes the value of the item at the point and the cost associated with the starved stations on the line due to the incompletion. Lau and Shtub (1987) suggest to stop the entire line only when certain tasks are not completed.

Although extensive research has been done on the deterministic version of the problem, relatively less work has been done to develop efficient, optimum-seeking solution procedures for the stochastic version. When task performance times are considered to be random variables, the sum of the performance times of the tasks assigned to a station may exceed $C$. Consequently, the enumeration and evaluation of the feasible sequences for the stochastic version of the problem is much more complex and time-consuming than the deterministic case. These characteristics of the stochastic version lead to the difficulty of developing an efficient optimum-seeking algorithm for this problem.

The stochasticity of the task performance times are recognized and stated by several authors, and most of them assumed normally distributed task performance times (Moodie and Young 1965, Mansoor and BenTuvia 1966, Kottas and Lau 1973, Reeve and Thomas 1973, Chakravarty and Shtub 1986, Silverman and Carter 1986, Vrat and Virani 1976, Wilhelm 1987, Yano and Bolat 1989), whereas there are some researchers assuming distributions other than normal for the performance times (Arcus 1966, Sphicas and Silverman 1976, Raouf and Tsui 1982).
3.1. Procedures to solve the stochastic assembly line balancing problem

With the relaxation of the deterministic task performance time assumption, several issues become relevant that complicate the analysis and development of solution procedures. We will examine the research conducted on the stochastic problem in three categories as follows:

(i) The first category involves the modified versions of the procedures developed for the SMD problem. There are two major formulations: the first formulation attempts to minimize labour cost subject to $S_{j} \leq a C$, for all $j$, where $0 \leq a \leq 1$. The second formulation also attempts to minimize labour cost, provided that at each station there is at least a given probability of completing the work within the given $C$. In the first formulation, the stochastic problem can be attacked with a deterministic problem procedure. On the other hand, the second formulation requires a procedure specifically designed to handle stochastic performance times. But, as Sphicas and Silverman (1976) state, the formulations are equivalent to each other for some task time distributions: these distributions are Poisson, gamma, binomial, negative binomial, chi-square and normal with parameters $\mu_{i}$ and $\sigma_{i}^{2}$ under the special condition that $s_{i}^{2}=k m_{i}$ for all $i$ where $k$ is a constant.

(ii) The second category involves the work performed to examine the problem characteristics via simulation, and to compare the deterministic and stochastic versions of the problem.

(iii) In the third category, there are procedures specifically developed for the stochastic problem.

The procedures in these categories are discussed in Sections 3.1.1, 3.1.2 and 3.1.3, respectively.

\subsubsection{M odified versions of the SMD problem procedures}

Moodie and Young (1965) modified their procedure developed for the deterministic problem to cope with the stochastic version. In order to deal with variable performance times, an allowance is provided to the operator for a given confidence level of task completion. Task performance times are assumed to be distributed normally. The station time of station $j$ is computed as:

$$
S_{j}=\sum_{i \in K_{j}} \mu_{i}+r \sqrt{\sum_{i \in K_{j}} \sigma_{i}^{2}}
$$


where $r$ is a constant multiplier and $K_{j}$ is the set of tasks assigned to station $j$. Assuming statistical independence between the tasks, the confidence level can be determined by the multiplier $r$. The procedure for the SMD problem transfers tasks among the stations with the objective of smoothing the differences between the stations times. The same procedure is utilized analogously for the SMS version: small and large variance tasks are transferred among the stations so that the variance summations are as nearly equal as possible for the stations. The authors neither suggest any means to determine the value of the multiplier, nor do they mention the relationship between the multiplier $r$ and the system cost. They do not consider the effect of incomplete units either. Although the procedure is computationally attractive, it performs poorly relative to the other procedures reported (Reeve and Thomas 1973, Vrat and Virani 1976, Koittas and Lau 1981).

Raouf and Tsui (1982), and Raouf et al. (1980) modified a procedure developed for the SMD problem by utilizing the theorem stated by Brady and Drury (1969). Assuming no correlation between the tasks, the theorem states that if a new task is added to a group of tasks, the coefficient of variation (CV) of the new station time will be less than that of the old station time, provided that the $\mathrm{CV}^{2}$ of the new task is less than $(1+2 * e) * \mathrm{CV}^{2}$ of the old station time, where $e$ is the ratio of the mean of the old station time to the mean of the new task performance time. The procedure does not assume normally distributed task performance times; instead, any known or unknown symmetrical distribution is accepted. If $P_{\mathrm{c}}$ denotes the probability that a station time can exceed $C$, then by utilizing Chebyshev's inequality, the station time for station $j$ can be expressed as follows:

$$
S_{j}=\sum_{i \in K_{j}} \mu_{i}+\sqrt{2 P_{\mathrm{c}} \sum_{i \in K_{j}} \sigma_{i}^{2}}
$$

The procedure first constructs the list of available tasks; these tasks are the ones with no unassigned predecessors in the precedence diagram. Then a set of tasks from the available list is formed by selecting the tasks which can be placed into the current station when the probability that the station time will exceed $C$ is less than a predetermined value. From this set of tasks, a subset is formed by selecting tasks such that its inclusion into the current station will satisfy the theorem of Brady and Drury (1969). If there is more than one task in this subset, then the one with the lowest CV is chosen. The authors conclude with a very limited experimentation that the procedure provides good and realistic results. More experimentation is required to test the performance of the procedure against the others reported in the literature and the behaviour of the procedure under various problem parameters.
Kao (1976) presented a DP approach in which the objective is to find a grouping of tasks that satisfies all precedence relations and minimizes the labour cost. The formulation is based on the argument that the probability of the resulting work content at each station not exceeding $C$ is bounded by a prespecified value. In other words, the goal is to assign tasks to a minimum number of stations, while observing all the precedence constraints and the constraint that, for all $j, \operatorname{Pr}\left(S_{j} \leq C\right) \geq \theta$, where $\theta$ is the given lower bound $(0 \leq \theta \leq 1)$. The task performance times are assumed to be random variables closed under convolution. When the task times are approximated by Poisson, gamma, binomial or negative binomial, the procedure results in the optimal solution. The procedure is useful only for problems of limited size due to the fact that storage and computation requirements grow very rapidly as the number of tasks increases. Kao (1979), and Kao and Queyranne (1982) later improved the computational aspects of the procedure in order to solve larger problems by utilizing a compact state labelling scheme, and better state generation procedures. With the improvements, the magnitude of the problems that can be solved still depends heavily on the problem parameters.

Shin (1990) developed a procedure that attempts to minimize the expected total cost which is the sum of labour cost and the cost arising from the incomplete tasks. Incomplete tasks are removed from the line and completed off the line. Thus, the downstream stations are kept idle for the corresponding cycle time. The procedure starts with a large $C$, and a balance is obtained by any deterministic heuristic procedure or algorithm. The expected total cost associated with the design is calculated. The cycle time is decremented by a fixed quantity and the procedure is repeated until $C$ is equal to the lower bound of $t_{\max }$. The balance associated with the minimum expected total cost constitutes the solution of the procedure.

Suresh and Sahu (1994) used simulated annealing to solve the SMS problem with the objectives of minimizing the smoothness index and the probability of stopping the line. Neighbour solutions are generated by transferring tasks among stations; this process is similar to the second phase of the procedure of Moodie and Young (1965). The performance of the procedure depends on the setting of various parameters, e.g. the rate of cooling and the number of configuration changes. The computation time is reported to increase exponentially as the cooling rate is decreased.

Suresh et al. (1996) presented two versions of GA to solve the SMS problem. Similar to the representation of Leu et al. (1994), feasible orderings of the tasks comprise the choromosomes. Smoothness index and the probability of stopping the line are utilized as the fitness function. 
In the first version, the feasibility of the offsprings is guaranteed by removing the duplicate tasks and the tasks with followers assigned to earlier stations. In the second version, two initial parent populations are utilized; one of which allows only feasible offsprings, while the other allows a fixed percentage of infeasible offsprings. Some specified members of these populations are exchanged. A few example problems are solved with up to 70 tasks, and the results are compared with the Trade-and-Transfer method of Reeve and Thomas (1973); the GA algorithm results in a significantly smaller probability of line stoppage for some problems. For example, in a 48-task problem, the probability of line stoppage turns out to be $0.3033,0.1474$ and 0.0924 in the trade-and-transfer method, the first and second versions of the GA algorithm, respectively.

\subsubsection{Simulation and comparative studies}

Reeve and Thomas (1973) compared four solution procedures for the SMS problem. In these procedures, an initial balance is given and the objective is to rearrange the tasks such that the probability that one or more station times exceed $C$ is minimized. The first procedure is based on the 'Trade and Transfer' concept introduced by Moodie and Young (1965). One-for-one task trades between stations are attempted in order to reduce the probability of exceeding $C$. The second procedure is the Branch-and-Bound technique, which utilizes the initial balance to establish upper bounds for the idle time and the probability of exceeding $C$. Then, lower bounds are calculated for each station alternative in terms of idle time and the probability of exceeding $C$. The procedure yields the optimal solution if carried to completion. The third procedure is the Heuristic Branch-and-Bound procedure, which is an extension of the previous one with some heuristic rules. The first rule utilizes a tighter lower bound by assuming the unassigned tasks being allocated to the empty stations such that these stations will have equal means and variances. The second rule divides the problem into subproblems. Then, the solutions of the subproblems are appended to each other. The last rule also divides the problem into subproblems which may be overlapping. The last procedure, BABTAB, is a combination of the first and third procedures. First the Heuristic Branch-and-Bound procedure is applied to the initial balance until no further improvements can be made. Then, the 'Trade-and-Transfer' procedure is applied to improve the design. They tested four problems and indicated that the Branch-and-Bound procedure guarantees a global optimum with excessive computer time. If an adequate supply of computer time is available, the Heuristic Branch-and-Bound procedure with the tighter lower bound and overlapping subproblems rule gives very good results. BABTAB yields good results in relatively short time periods. The Trade-and-Transfer procedure is the least effective one. On the other hand, the conclusions reached are not justified and cannot be generalized since the number of example problems solved is very small.

Buxey et al. (1973) examined SMS assembly lines via Monte Carlo simulation and determined that on-line inventory is vital to ensure efficient performance, and for good line designs, and the ratio of on-line inventory to number of stations should be greater than unity. The simulation study also reveals that a criterion of maximizing output would imply the acceptance of a small proportion of unfinished units. The level of unfinished units being carried should be in the region of 1 or $5 \%$, and should never be as high as $10 \%$.

Driscoll and Abdel-Shafi (1985) developed a balancing procedure linked with a simulation study to evaluate the performance of the solutions under changing conditions. The balancing procedure is similar to the RPWT developed by Helgeson and Birnie (1961). The stochasticity of task performance times is treated similar to the approach of Moodie and Young (1965). Two other procedures are also developed for mixed-model lines. The performance of the lines is examined with changes in the following parameters: line speed, physical make-up of stations and product mix for the mixed-model lines. Both balance delay and smoothness index are reduced with increases in line speed. On the other hand, the amount of incompletions increases as the line is speeded up. The physical make-up of stations can vary as the station turns into an open station from a closed one. The simulation model indicates that with open stations, the operators tend to move out of their normal working zones. This raises questions about the introduction of inefficient working practices. The concept of evaluating line designs under various conditions is a very useful one, since the conditions hardly remain the same after the design has been made. The sensitivity of the design to those changes may help researchers to develop more efficient heuristic procedures.

Moberly and Wyman (1973) examined the means to increase output rate with a simulation study. They attempted to answer the following question: if there exist alternatives in constructing two single independent lines or one dual line (or expedited dual line) at the same cost, which of these configurations should be selected to give the maximum output rate? In a dual line, a station of the ordinary line is replaced by two identical parallel stations. In an expedited dual line, a station opposite to a failed (with incompletions) station has twice the service rate that it normally has. Station times are assumed to be normally distributed with a mean of 1 and standard devi- 
ation of 0.3 for all stations. In other words, it is assumed that the lines are well-balanced and the CV of the station times is 0.3 for all stations. The analysis indicates that it can be too costly to increase output rate by increasing the line length (or increasing $C$ ) due to the resulting imbalance. It is advisable to consider constructing a dual line rather than another single line. Furthermore, a dual line has an added advantage of possibly becoming an expedited dual line.

Arcus (1966) suggested to allocate some idle time to the workers to compensate for the effects of stochastic performance. Station times are multiplied by a weight randomly generated from a binomial distribution. Station times are converted into distances moved by assuming an unpaced assembly line. The stimulation of the line with the new station times results in the expansion of the stations down the line. With this expected result, it can be concluded that if the line is sufficiently long, the problem is solved. Otherwise, there are the alternatives of reducing production rate, lengthening the line, working off the line, or employing extra workers roving along the line and helping the line workers.

Silverman and Carter (1986) examined the effect of stochastic task performance times on the total operating cost of a line in which the incompletions are handled by stopping the entire line for the time necessary to complete the work. The line balancing procedure is quite similar to the procedure of Arcus (1966). Tasks with no unassigned predecessors are assigned to stations subject to the constraint that the probability of exceeding $C$ is below a specified value. Tasks are selected for assignment randomly, whereas Arcus (1966) utilizes several biasing rules for selection. The designs generated are evaluated with the following cost function:

$$
E(T C)=(C \times L \times K)+L_{\mathrm{a}} \int_{C}^{\infty}(1-G(w)) \cdot \mathrm{d} w
$$

where $L$ is the labour rate, $L_{\mathrm{a}}$ is the overtime labour rate, $w$ is the maximum time required for all stations to complete their tasks in a particular cycle and $G(w)$ is the corresponding cumulative distribution function. Note that the cost of not being able to complete all tasks within $C$ is calculated at the overtime rate. The design with the lowest $E(T C)$ value is retained as the 'best'. This design is compared with the results of two procedures in which tasks are assigned to stations as long as a fixed percent of $C$ is not exceeded. In almost all the problems solved, the procedure performs better; the savings are significantly greater for problems with higher overtime rates. Results should be compared with the other more effective procedures reported in the literature to generalize on the performance of the procedure.

\subsubsection{Procedures developed solely for the stochastic problem}

None of the procedures described above, with the exceptions of Silverman and Carter (1986), and Shin (1990), consider the cost term arising from the incomplete tasks. Instead, the procedures attempt to minimize the cost term by allocating some slack to stations or by ensuring that the probability of completion at each station is less than a specified value. But, $C$ may be uneconomically high before the incompletions become negligible. On the other hand, for realistic ranges of $C$, the incompletions and associated costs are far from negligible. There is a trade-off between the cost of idle time at a station and the cost of incompletion. Thus, the $I C$ arising from the incomplete tasks and the labour cost both should be considered simultaneously. The procedures in this section adopt the following objective function:

$$
\begin{aligned}
\text { Min, } T C= & \text { Total Labour Cost } \\
& + \text { Total Expected Incompletion Cost }
\end{aligned}
$$

For a given $K$, the computation of the total labour cost term is straightforward; it is linearly proportional to $K$, and is equal to $K \times L \times C$. On the other hand, the computation of the total expected incompletion cost (EIC) term is much more complex and involves the computations of several other variables. For a given $K$ and allocations of tasks to these stations, the following variables should be determined for each task in order to compute the total EIC term: (i) the probability that the task can start to be processed; (ii) the probability that the task is not completed within $C$ after it has started to be processed; and (iii) the cost incurred when the task is not completed within $C$. Note that the incompletions are assumed to be completed off the line. A detailed discussion, and the derivation of the variables and a general expression which captures the cost factors are given by Sarin and Erel (1990).

Kottas and Lau (1973) developed a heuristic procedure which attempts to minimize the above cost function. Whenever a task is not finished, the unit goes down the line with as many of the remaining tasks being completed as possible. All unfinished tasks are completed off the line; the cost to complete the task off the line is not a function of what fraction of the task is completed on the line. Their procedure can be described as follows: an available list is formed by identifying the tasks with no unassigned predecessors. This list is updated each time a task is assigned. Then, a desirable list is formed by identifying the available list tasks which are marginally desirable for assignment. A task is considered marginally desirable when its anticipated labour savings in the specific position under consideration are larger than its EIC. 
The tasks with virtual certainty of completion are assigned first in descending order of their ICS. These tasks comprise the sure list. If the sure list is empty, then the desirable list tasks are assigned in the ascending order to their ICS. When the desirable list gets empty, a new station is established. The tasks which are never marginally desirable are assigned as the first tasks in stations as soon as they are placed in the available list. The procedure continues until the available list gets empty. The procedure is computationally very attractive; however, since the procedure is a single-pass technique, the solution found might be far from the optimal solution. Kottas and Lau (1976) later developed a procedure for evaluating the EIC of a design. The procedure identifies all the possible combinations of incomplete tasks, and for each combination, the occurrence probabilities and associated costs are calculated.

Kottas and Lau (1981) developed another heuristic procedure which generates several promising line designs. The procedure is an extension of their earlier work (1973); several selection rules and their probabilistic combinations are utilized for the tasks in the desirable list. The selection process is conceptually related to the 'weighted random selection method' of Arcus (1966) and the selection by 'probabilistic combinations of heuristics' of Tonge (1965). The selection rules utilized include the probabilistic combinations of the following rules: random selection, select task with the largest $I C$, select task with the lowest $I C$, select task with the highest $\mu_{i}$, and select task with the highest value of $\mu_{i} / I C_{i}$. Thus, several line designs are generated and the dominated designs are eliminated. The remaining ones are evaluated with their evaluation procedure (Kottas and Lau 1976). The design with the lowest cost is the solution to the procedure. The performance of the procedure is compared with the procedure of Moodie and Young (1965), in which the confidence level of total completion in each station varies from 80 to $98 \%$. In the limited experimentation reported, the procedure results in lower-cost designs for the 24 problems solved; the average saving is $4 \%$.

Vrat and Virani (1976) applied Kottas and Lau's (1973) technique to a real life problem with 51 stations and 75 workers, some of which are working outside the line. They modified the technique to enable it to tackle task times greater than $C$ by paralleling of stations for such tasks. The number of parallel stations required for task $i$ is $\left[\mu_{i} / C\right]$. If some slack time remains in the equivalent single station, then other tasks(s) may be assigned if precedence restrictions allow. Virtual task time is defined as the difference between $C$ and the slack. The procedure continues with all task times being compared with the corresponding virtual task times. The procedure is also modified to allow mixed-model lines as follows. Past data are utilized to determine the proportional occurrences of the model variations, which form the weight factor to be used for calculating $\mu_{i} \mathrm{~s}$ and $\sigma_{i}^{2} \mathrm{~s}$. The results obtained for different $C$ values are compared with those obtained using the procedure of Moodie and Young (1965) for $95 \%$ or more probability of completion at each station. For almost all values of $C$, the procedure results in designs which require a lower number of operators for manning the line, give smaller smoothness indices, and lower total operating costs.

Shtub (1984) presented an heuristic procedure for designing lines with stochastic task times and multiple manning of stations. The mean duration time of a task is assumed to be a non-increasing discrete function of the number of workers at the station to which the task is assigned. The procedure is quite similar to Kottas and Lau's (1973) technique. Formation of the available and fit (desirable) list is almost identical; the desirability of a task is determined considering the number of workers assigned to the station. When the fit list gets empty, an estimate of the number of subsequent stations is made using the technique of Kottas and Lau (1973) assuming that each of the subsequent stations is manned by its maximum work force. If the estimated total number of stations is larger than a critical value, the current station is reopened with the work force set to the next highest possible value. Otherwise, the next station is opened with the minimum possible work force.

Sculli (1979) considered adjusting the line design after the initial design is obtained, since several dynamic factors are involved in the process: $C$ may have to be adjusted depending on the value of $D$, changes in specification occur and operators do not work at standard rates. Later, Sculli (1984) developed a computer program which finds a compromise solution to the line balancing problem by interacting with the user. The constraints involved in the procedure are that the output from a station should be sufficient to keep the following station working, and the number of workers assigned to a station must not exceed a given upper bound. The program attempts to assign workers in an optimal manner; if a solution is not possible, options to change output rate or other constraints are offered, so that a compromise solution could be found. The program does not have any academic interest, but has a practical value because it is intended for use by the line manager to determine the allocation of operators at the start of each shift or work period' (Sculli 1984).

Sarin et al. (1997) developed an enumeration-based approximation methodology that makes use of some insights and effective solutions to subproblems of the SMS problem. The objective function comprises the total $L C$ and total EIC. A general expression is developed in Sarin and Erel (1990) that captures the cost terms of 
the objective function given the allocation and ordering of the tasks to a given $K$. The expression of the objective function is as follows:

$\operatorname{Min} Z-(C \times L \times K)+\sum_{i=1}^{N}\left[\beta_{i}\left(I C_{i}+\sum_{k \in \beta_{i}} I C_{k}\right)-\sum_{j=1}^{f s_{i}} S B_{i}^{j}\right]$

where $\beta_{i}$ is the probability that task $i$ has started to be processed and not completed within $C, B_{i}$ is the set of tasks which cannot be started and therefore are incomplete due to the incompletion of task $i, f s_{i}$ is the total number of starting events of task $i$ (i.e. events that can lead to the starting of task $i$ ), and $S B_{i}^{j}$ is a correction factor for the overcounted incompletion costs corresponding to starting event $j$. The process of computing relevant incompletion probabilities and costs is achieved with an enumeration tree in which all possible ways of realizing the starting events are represented with their occurrence probabilities. The proposed procedure divides the problem into subproblems and obtains an initial solution to each subproblem using a DP procedure with a bounding strategy. Then, the initial solutions are improved with a branch-and-bound type of procedure. Finally, the improved solutions are appended to each other to obtain the final solution of the problem. The experimental results indicate that the procedure generates better solutions than those obtained by the technique of Kottas and Lau (1981) with reasonable computation requirements.

Lau and Shtub (1987) proposed the concept of hybrid line in which the cycle time can be extended occasionally when some task incompletions occur. Tasks are divided into two classes: the line is stopped for as long as needed for the completion of the tasks in the first class, whereas the line is not stopped even if the tasks in the second class are not completed within the 'base cycle time'. The concept introduced by the authors is an interesting one, although several issues remain open, e.g. the classification of the tasks into the two classes.

\subsection{Procedures to solve the M MS problem}

Significantly less research has been conducted for the MMS problem compared to the other versions. This situation is contributed to the increased complexity of the problem. There are a few researchers who modified their procedures developed for single-model versions to handle the mixed-model version (Vrat and Virani 1976).

Johnson (1983) developed a branch-and-bound algorithm for the SMD problem. He has also pointed out some modifications that can be easily implemented into his procedure. Stochasticity of the task times, requirement of task groupings according to task skill levels, and mixed-model situations are among the modifications considered.

Arcus (1966) also considered stochasticity of the tasks times and mixed-model situations as the modifications for COMSOAL. But, for the mixed-model situation, he assumed deterministic task times.

Chakravarty and Shtub (1986) developed two heuristic procedures for mixed-model stochastic assembly lines in which different models of the same general product are assembled. The approach allows for in-process inventories between stations. The objective is to minimize the total operating cost, composed of labour cost, in-process inventory cost and setup cost, subject to two constraints, i.e. precedence relations among tasks and that the probability of completing the set of tasks assigned to any station is not less than a specified value. The first procedure is a single-pass heuristic which is based on the concept of 'positional weights' developed by Helgeson and Birnie (1961). The second procedure is a shortestpath heuristic which is based on the consecutive sequencing of the tasks. Both of the procedures are highly sensitive to the precedence structure of the problem; more effort is required as the $F$-ratio increases. The first procedure has an advantage over the shortest-path procedure in the CPU time requirement, but the latter always performs better.

\section{Evaluation and concluding remarks}

The ALBP is a problem of practical interest and has gained the attention of numerous researchers who developed various solution procedures and applied them to various environments including the service industry (Levary and Renfro 1991). The problem is NP-hard, since a special version of the problem, i.e. the one involving no precedence relations among tasks is a bin-packing problem which is NP-hard in the strong sense. Faaland et al. (1992) state that the existing procedures attempting to find the optimal solution have a complexity of at least $2^{N}$. Hence, developing heuristic procedures to solve problems of a practical size remains the only option for the researchers. The optimum-seeking algorithms can only be used to evaluate the performance of the heuristic procedures by providing the optimal solutions to the problems of restricted sizes.

Kim and Park (1995) state that another motivation to develop heuristic procedures is that the task times utilized in these procedures are usually rough estimates which do not account for the variability between workers and throughout time; thus, obtaining a 'good' solution rather than the optimal one may be acceptable to the practitioners. Another source of difficulty in obtaining accurate estimates for the task times is the learning phenomenon. 
Thus, we also agree with Kim and Park (1995) that an efficient and reasonable heuristic procedure would be sufficient to satisfy the needs of practitioners. But, we must also note that even a small change in design parameters may lead to significant savings or losses in production costs and other performance measures. Hence, one must be very careful in selecting the procedure to be utilized.

Wee and Magazine (1982) have presented a worst-case performance analysis of the heuristic procedures for the deterministic ALBP. Let $K_{\text {heu }}$ and $K_{\text {opt }}$ be the number of stations found using some heuristic procedure and the optimal number of stations, respectively. They have shown that $K_{\text {heu }} \leq 2 K_{\text {opt }}-1$. As given in Queyranne (1985), the authors, in an earlier working paper, also showed that $K_{\text {heu }}<K_{\text {opt }} /\left(1-t_{\max } / C\right)+1$ if the heuristic procedure is reasonable; a heuristic procedure is reasonable if $S_{j}>C-t_{\max }$ for $j=1, \ldots,\left(K_{\text {heu }}-1\right)$. Queyranne $(1985)$ has complemented the above results by showing that no polynomial heuristic can have a worst-case ratio less than 1.5 , unless $P=N P$. In other words, all polynomial heuristic procedures must have a worst-case performance ratio in the interval $[1.5 ; 2]$. Although the worst-case performance analysis (unlike the average-case analysis, empirical or theoretical) may not have as much value to a practitioner, the above bounds can still act as useful benchmarks and have significant academic value.

For the SMD version of the problem, the existing heuristic procedures, most of the time, provide near-optimal, if not optimal, solutions to the problems of realistic sizes within reasonable computation time. Multi-pass and backtracking procedures are expected to outperform the single-pass procedures. However, even the procedure of Baybars (1986a), a single-pass procedure applied to the simplified and reduced problem, is also reported to find, in most cases, the optimal solution of problems with up to 83 tasks within negligible computation time. Thus, we can safely conclude that the existing heuristic procedures are sufficient to satisfy the needs of line designers in industry.

For the MMD version of the problem, the integer programming formulations (Roberts and Villa 1970, Gokcen and Erel 1997) are far from being applicable to problems of realistic sizes due to the excessive storage and computation requirements. However, several heuristic procedures exist that give 'good quality' solutions.

The amount of research conducted on the stochastic version of the problem is much smaller due to the complex nature of the problem. For the MMS version of the problem, we have found only one procedure in the open literature (Chakravarty and Shtub 1986).

In this paper, we have presented a detailed review of the heuristic procedures developed for the SMD problem, and the procedures developed for the other versions of the problem. The most recent survey of the subject has been conducted by Ghosh and Gagnon (1989); the studies that have been published in the open literature after the survey of Ghosh and Gagnon (1989) are listed in table 2 with some basic information on the approaches. Considering the large number of studies reported in the literature, it is interesting to note that developing procedures for the SMD version of the problem still continues to be an active research area.

Although numerous articles have been published that develop optimum-seeking or heuristic procedures for the solution of ALBPs, the procedures attempting to solve the stochastic and mixed/multi model versions of the problem are far from adequate to satisfy the needs of industry. For the SMD version, the available procedures can be considered partially satisfactory with respect to the problem sizes and the flexibility to model the actual conditions of assembly lines. Thus, developing procedures for the solution of SMS, MMD and MMS versions of the problem that are both capable of solving problem sizes and flexible to model the actual conditions encountered in practice constitutes a future research area. We also point out below some design issues that need to be studied further.

(1) An issue that may have a significant impact on the design of assembly lines is the learning phenomenon. Having different learning rates for the tasks assigned to different stations will distort the work allocation of even the perfectly balanced lines. In mixed/multi model lines, the forgetting effect further complicates the problem. Chakravarty and Shtub (1992) developed a non-linear, integer model for the MMD version in which the objective is to minimize the inventory holding, setup and labour costs subject to the constraint of decreasing station times as the operators gain experience. The authors solve the model with a heuristic procedure called 'Branch-and-Estimate Procedure'. Developing procedures that consider the new developments in the learning and the forgetting effect for the mixed/multi model lines, and conducting studies that examine the effects of these phenomena on the line balance are further research areas.

(2) In SMS and MMS versions of the problem, the majority of the studies assume the handling of task incompletions as follows: the units go down the line with as many of the remaining tasks being completed as possible and the remaining tasks are completed off the line (Kottas and Lau 1973, 1976, Vrat and Virani 1976, Kottas and Lau 1981, Sarin and Erel 1990, Sarin et al. 1997). Other approaches to 
Table 2. Recent research conducted on assembly line balancing problems.

\begin{tabular}{llll}
\hline \multicolumn{1}{c}{ Reference } & Year & $\begin{array}{c}\text { Problem } \\
\text { version }\end{array}$ & \\
\hline Agrawal & 1985 & SMD & Single-pass similar to RPWT \\
Anderson and Ferris & 1994 & SMD & Genetic algorithm \\
Berger et al. & 1992 & MMD & Branch-and-bound with truncated search \\
Buxey & 1978 & SMS & Monte Carlo simulation \\
Chakravarty and Shrub & 1986 & MMS & Single-pass and a shortest-path procedure \\
Driscoll and Abdel-Shafi & 1985 & MMS & Single-pass linked with a simulation model \\
Easton & 1990 & SMD & DP with relaxation and fathoming \\
Faaland et al. & 1992 & SMD & Heuristic shortest-path \\
Gokcen and Erel & 1997 & MMD & Binary integer programming model \\
Hackman et al. & 1989 & SMD & Branch-and-bound algorithm with heuristic fathoming rules \\
Hoffmann & 1990 & SMD & An enumeration procedure based on Hoffmann's heuristic [35] \\
Kim and Park & 1995 & SMD & Cutting plane algorithm \\
Lau and Shtub & 1987 & SMS & Procedure based on a hybrid line concept \\
Leu et al. & 1994 & SMD & Genetic algorithm \\
Miltenburg and Wijngaard & 1994 & SMD & DP and heuristic for U-line problem \\
Nkasu and Leung & 1995 & SMS & COMSOAL-based procedure \\
Raouf and Tsui & 1982 & SMS & Priority-ranking heuristic \\
Sarin and Erel & 1990 & SMS & Heuristic dynamic programming \\
Sarin et al. & 1997 & SMS & Approximate procedure based on heuristic DP \\
Scholl and Voß & 1996 & SMD & Priority-ranking heuristic and tabu search \\
Sculli & 1984 & SMS & Priority-ranking heuristic \\
Shin & 1990 & SMS & A procedure that can use any heuristic or exact method in the literature \\
Shtub & 1984 & SMS & Priority-ranking heuristic \\
Shtub and Dar-El & 1990 & SMD & Procedure utilizing assembly chart information and MALB \\
Silverman and Carter & 1986 & SMS & Mutli-pass procedure \\
Sparling and Miltenburg & 1997 & MMD & Heuristic for U-line problem \\
Suresh and Sahu & 1994 & SMS & Simulated annealing \\
Suresh et al. & 1996 & SMS & Genetic algorithm \\
Yano and Bolat & 1989 & SMD & Heuristic branch-and-bound procedure \\
\hline
\end{tabular}

handling task incompletions, e.g. scraping the unit when an incompletion occurs, locating special stations along the line that complete the missing tasks, or stopping the whole line (Silverman and Carter 1986) should also be considered. The 'hybrid line' concept suggested by Lau and Shtub (1987) seems to have the potential of generating designs with lower total costs and needs to be further studied.

(3) Employing buffer capacity between stations in lines with stochastic task times may improve the performance of these lines by providing partially completed units to stations downstream of the station facing task incompletions. On the other hand, the performance increase should be compared with the various cost factors associated with utilizing buffer capacities between stations. Buffer capacity utilization in paced assembly lines is another area that needs to be further studied.

(4) As also stated by Ghosh and Gagnon (1989), an industrial survey that reveals the current practices of line designers should be conducted. The most recent survey dates back to Chase (1974); the impacts of the fast development in the computer hardware and software, and the significant change in quality management and human resources management on the assembly lines should be questioned in these two decades. The survey should also assess the needs of the practitioners to help the academicians to construct models and develop solution procedures.

(5) A comparative study that evaluates the performance of the recently developed procedures with respect to the solution quality and computational requirements needs to be conducted. Such a study would enable the practitioners to choose the most appropriate procedures for their needs, as well as guiding the academicians to improve the current procedures and develop more effective ones.

(6) Recently, many assembly lines are designed as $\mathrm{U}$-shaped that allow more flexibility in grouping tasks into stations. Miltenburg and Wijngaard (1994) introduced the U-line balancing problem for the SMD version and developed three procedures; the first is an optimum-seeking DP formu- 
lation based on the formulation of Held et al. (1963), and the other two are heuristic procedures based on the RPWT of Helgeson and Birnie (1961) and the enumeration procedure of Hoffmann (1963). Sparling and Mittenburg (1997) address the U-line problem for the MMD vesion and present an approximate procedure. Several directions for future research on the U-line balancing problem exist, including studying SMS lines and considering various new objectives.

\section{References}

Agrawal, P. K., 1985, The related activity concept in assembly line balancing. International J ournal of Production Research, 23, 403-421.

Akagi, F., Osaki, H., and Kikichi, S., 1983, A method for assembly line balancing with more than one worker in each station. International J ournal of Production Research, 21, 755-770.

Anderson, E. J., and FERris, M. C., 1994, Genetic algorithms for combinatorial optimization: the assembly line balancing problem. ORSA J ournal on Computing, 6, 161-173.

Arcus, A. L., 1966, COMSOAL: A computer method of sequencing operations for assembly lines. International J ournal of Production Research, 4, 259-277.

BAYBARs, I., 1986a, An efficient heuristic method for the simple assembly line balancing problem. International I ournal of Production Research, 24, 149-166.

BAYBARs, I., 1986b, A survey of exact algorithms for the simple assembly line balancing problem. Management Science, 32, 909-932.

BennetT, G. B., and ByRd, J., 1976, A trainable heuristic procedure for the assembly line balancing problem. AIIE Transactions, 8, 195-201.

Berger, I., Bourjolly, J. M., and Laporte, G., 1992, Branch-and-bound algorithms for the multi-product assembly line balancing problem. European J ournal of Operational Research, 58, 215-222.

Bowman, E. H., 1960, Assembly-line balancing by linear programming. Operations Research, 8, 385-389.

Brady, W., and Drury, C. G., 1969, The dependence of the coefficient of variation of a work time distribution of a number of elements in the work task. International J ournal of Production Research, 7, 311.

Buxey, G., 1978, Incompletion costs versus labour efficiency on the fixed-item moving belt flowline. International J ournal of Production Research, 16, 233-247.

Buxey, G. M., Slack, N. D., and WILd, R, 1973, Production flow line system design - a review. AIIE T ransactions, 5, 37-48.

Chakravarty, A. K, and Shtub, A., 1985, Balancing mixed model lines with in-process inventories. M anagement Science, 31, 1161-1174.

Chakravarty, A. K. and Shtub, A., 1986, A cost minimization procedure for mixed model production lines with normally distributed task times. European J ournal of Operational Research, 23, 25-36.

Chakravarty, A. K, and Shrub, A., 1992, The effect of learning on the operation of mixed-model assembly lines. Production and Operations M anagement, 1, 198-211.
Chase, R. B., 1974, Survey of paced assembly lines. Industrial Engineering, 6, 14-18.

Dallery, Y., and Gershwin, S. B., 1992, Manufacturing flow line systems: a review of models and analytical results. Queuing Systems, 12, 3-94.

DAR-EL, E. M., 1973, MALB-a heuristic technique for balancing large single-model assembly lines. AIIE T ransactions, 5, 343-356.

DAR-EL, E. M., 1975, Solving large single-model assembly line balancing problems - a comparative study. AIIE T ransactions, 7, 302-310.

DAR-EL, E. M., 1978, Mixed-model assembly line sequencing problems. OMEGA, 6, 313-323.

DAR-EL, E. M., and Cother, R. F., 1975, Assembly line sequencing for model mix. International J ournal of Production Research, 13, 463-477.

DAR-EL, E. M, and Cucuy, S., 1977, Optimal mixed-model sequencing for balanced assembly lines. OM E GA, 5, 333-342.

DAR-EL, E. M., and Nadivi, A., 1981, A mixed-model sequencing application. International J ournal of Production Research, 19, 69-84.

DAR-EL, E. M., and RuBinovitch, Y., 1979, MUST-a multiple solutions technique for balancing single model assembly lines. M anagement Science, 25, 1105-1114.

Driscoll, J., and AbDel-Shafi, A. A. A., 1985, A simulation approach to evaluating assembly line balancing solutions. International J ournal of Production Research, 23, 975-985.

Easton, F. F., 1990, A dynamic program with fathoming and dynamic upper bounds for the assembly line balancing problem. Computers and Operations Research, 17, 163-175.

Faaland, B. H., Klastorin, T. D., Schmitt, T. G., and Shtub, A, 1992, Assembly line balancing with resource dependent task times. Decision Sciences, 23, 343-364.

Gehrlein, W. V., and Patterson, J. H., 1978, Balancing single-model assembly lines: Comments on a paper by E. M. Dar-El (Mansoor). AIIE T ransactions, 10, 109-112.

GHosh, S., and GaGnon, R. J., 1989, A comprehensive literature review and analysis of the design, balancing and scheduling of assembly systems. International J ournal of Production Research, 27, 637-670.

Gokcen, H., and EREL, E., 1997, Binary integer formulation for mixed-model assembly line balancing problem. Computers and Industrial E ngineering, to appear.

Gutjahr, A. L., and NeMHauser, G. L., 1964, An algorithm for the line balancing problem. $M$ anagement Science, 11, 308315.

Hackian, S. T., Magazine, M.J., and Wee, T. S., 1989, Fast, effective algorithms for simple assembly line balancing problems. Operations Research, 37, 916-924.

Held, M., Karp, R. M, and Shareshian, R, 1963, Assemblyline balancing-Dynamic programming with precedence constraints. Operations Research, 11, 442-459.

Helgeson, W. B, and BIRNIe, D. P., 1961, Assembly line balancing using the ranked positional weight technique. J ournal of Industrial Engineering, 12, 394-398.

Hoffmann, T. R., 1963, Assembly line balancing with a precedence matrix. $M$ anagement Science, 9, 551-562.

HofFuann, T. R, 1990, Assembly line balancing: a set of challenging problems. International J ournal of Production Research, 28, 1807-1815.

Hu, T. C., 1961, Parallel sequencing and assembly line problems. Operations Research, 9, 841-848.

IGNALL, E. J., 1965, A review of assembly line balancing. $J$ ournal of Industrial E ngineering, 16, 244-254. 
Jackson, J. R, 1956, A computing procedure for a line balancing problem. $M$ anagement Science, 3, 261-271.

Johnson, R. V., 1981, Assembly line balancing algorithms: computation comparisons. International J ournal of Production Research, 19, 277-287.

Johnson, R. V., 1983, A branch and bound algorithm for assembly line balancing problems with formulation irregularities. M anagement Science, 29, 1309-1324.

KaO, E. P. C., 1976, A preference order dynamic program for stochastic assembly line balancing. M anagement Science, 22, 1097-1104.

KaO, E. P. C., 1997, Computational experience with a stochastic assembly line balancing algorithm. Computers and Operations Research, 6, 79-86.

KaO, E. P. C., and Queyranne, M., 1982, On dynamic programming methods for assembly line balancing. Operations Research, 30, 375-390.

KILBRIDGe, M. D., and Wester, L., 1961a, The balance delay problem. M anagement Science, 8, 69-84.

KIlbridge, M. D., and Wester, L., 1961b, A heuristic method of assembly line balancing. J ournal of Industrial E ngineering, 12, 292-298.

KILBRIDGe, M. D., and Wester, L., 1962a, Heuristic line balancing: A case. J ournal of Industrial Engineering, 13, 139-149.

Kilbridge, M. D., and Wester, L., 1962b, A review of analytical systems of line balancing. Operations Research, 10, 626638.

KIM H., and PARK, S., 1995, A strong cutting plane algorithm for the robotic assembly line balancing problem. International J ournal of Production Research, 33, 2311-2323.

KotTas, J. F., and Lau, H. S., 1973, A cost oriented approach to stochastic line balancing. AIIE T ransactions, 5, 164-171.

Kottas, J. F., and Lau, H. S., 1976, A total operating cost model for paced lines with stochastic task times. AIIE Transactions, 8, 234-240.

Kottas, J. F., and Lau, H. S., 1981, A stochastic line balancing procedure. International J ournal of Production Research, 19, 177193.

Kwo, T. T., 1958, A theory of conveyors. $M$ anagement Science, 5, $51-71$.

Lau, H. S., and Shrub, A., 1987, A exploratory study on stopping a paced line when incompletions occur. IEE Transactions, 19, 463-467.

Leu, Y. Y., Matheson, L. A., and Rees, L. P., 1994, Assembly line balancing using genetic algorithms with heuristic-generated initial populations and multiple evaluation criteria. Decision Sciences, 25, 581-606.

Levary, R. R, and Renfro, M. D., 1991, Application of assembly line balancing techniques to installment lending operations of commercial banks. Computers and Industrial E ngineering, 20, 105-109.

Mansoor, E. M, and Ben-Tuvia, S., 1966, Optimizing balanced assembly lines. J ournal of Industrial E ngineering, 17, $126-131$.

Mastor, A. A, 1970, An experimental investigation and comparative evaluation of production line balancing techniques. $M$ anagement Science, 16, 728-746.

MiLas, G., 1990, Assembly line balancing. . . Let's remove the mystery. Industrial E ngineering, 22, 31-36.

Miltenburg, G. J., and Wijngaard, J., 1994, The U-line line balancing problem. M anagement Science, 40, 1378-1388.

Moberly, L. E., and Wyman, F. P., 1973, An application of simulation to the comparison of assembly line configurations. Decision Sciences, 4, 505-516.
Moodie, C. L., and Young, H. H., 1965, A heuristic method of assembly line balancing for assumptions of constant or variable work element times. J ournal of Industrial Engineering, 16, 23-29.

Nevins, A. J., 1972, Assembly line balancing using best bud search. M anagement Science, 18, 529-539.

Nkasu, M. M., and Leung, K. H., 1995, A stochastic approach to assembly line balancing. International J ournal of Production Research, 33, 975-991.

Pinto, P. A., Dannenbring, D. G., and Khumawala, B. M, 1978, A heuristic network procedure for the assembly line balancing problem. Naval Research Logistics Review, 25, 299307.

Prenting, T. O., and Battaglin, R. M., 1964, The precedence diagram: a tool for analysis in assembly line balancing. $J$ ournal of Industrial E ngineering, 15, 208-213.

Queyranne, M., 1985, Bounds for assembly line balancing heuristics. Operations Research, 33, 1353-1359.

Raouf, A., El-SAyed, E, A., and Tsu, C. L., 1980, A new heuristic approach to assembly line balancing. Computers and Industrial Engineering, 4, 223-234.

Raouf, A., and Tsui, C. L., 1982, A new method for assembly line balancing having stochastic work elements. Computers and Industrial Engineering, 6, 131-148.

Reeve, N. R, and Thomas, W. H., 1973, Balancing stochastic assembly lines. AIIE Transactions, 5, 223-229.

Roberts, S. D., and VILLA, C. D., 1970, On a multiproduct assembly line-balancing problem. AIIE Transactions, $2,361-$ 364.

Rosenblatt, M. J., and Carlson, R. C., 1985, Designing a production line to maximize profit. IIE T ransactions, 17, 117121.

Salveson, M. E., 1955, The assembly line balancing problem. J ournal of Industrial E ngineering, 6, 18-25.

SARIN, S. C., and ERel, E., 1990, Development of cost model for the single-model stochastic assembly line balancing problem. International J ournal of Production Research, 28, 1305-1316.

SARIN, S. C., EREL, E., and DAR-EL, E. M., 1997, A methodology for solving single-model, stochastic assembly line balancing problem. Working paper.

SARker, B. R., and Shanthikumar, J. G., 1983, A generalized approach for serial or parallel line balancing. International J ournal of Production Research, 21, 109-133.

Schofield, N. A., 1979, Assembly line balancing and the application of computer techniques. Computers and Industrial Engineering, 3, 53-59.

Scholl, A., and Voss, S., 1996, Simple assembly line balancing-heuristic approaches. J ournal of Heuristics, 2, 217-244.

Sculli, D., 1979, Dynamic aspects of line balancing. OM E GA, 7, 557-561.

Sculli, D., 1984, Short term adjustments to production lines. Computers and Industrial E ngineering, 8, 53-63.

SHIN, D., 1990, An efficient heuristic for solving stochastic assembly line balancing problems. Computers and Industrial E ngineering, 18, 285-295.

ShtuB, A, 1984, The effect of incompletion cost on the line balancing with multiple manning of work stations. International J ournal of Production Research, 22, 235-245.

Shtub, A., and Dar-EL, E. M., 1990, An assembly chart oriented assembly line balancing approach. International J ournal of Production Research, 28, 1137-1151.

Silverman, F. N., and Carter, J. C., 1986, A cost-based methodology for stochastic line balancing with intermittent line stoppages. M anagement Science, 32, 455-463. 
Smunt, T. L., and Perkins, W. C., 1985, Stochastic unpaced line design: review and further experimental results. $J$ ournal of Operations $M$ anagement, 5, 351-373.

Sparling, D., and Miltenburg, J., 1998, The mixed-model U-line balancing problem. International J ournal of Production Research, 36, 485-501.

Sphicas, G. P., and Silverman, F. N., 1976, Deterministic equivalents for stochastic assembly line balancing. AIIE Transactions, 8, 280-282.

SuPnick, F., and Solinger, J., 1960, An external productionline problem. Operations Research, 8, 381-384.

Suresh, G., and SAHU, S., 1994, Stochastic assembly line balancing using simulated annealing. International J ournal of Production Research, 32, 1801-1810.

Suresh, G., VINOD, V. V., and SAhU, S., 1996, A genetic algorithm for assembly line balancing. Production Planning \& Control, 7, 38-46.

Talbot, F. B., Patterson, J. H., and Gehrlein, W. V., 1986, A comparative evaluation of heuristic line balancing techniques. M anagement Science, 32, 430-454.

Thomopoulos, N. T., 1967, Line balancing-sequencing for mixed-model assembly. $M$ anagement Science, 14, B59-B75.

Thomopoulos, N. T., 1970, Mixed model line balancing with smoothed station assignments. M anagement Science, 16, 593-603.

Tonge, F. M., 1960, Summary of a heuristic line balancing procedure. M anagement Science, 7, 21-42.

Tonge, F. M., 1961, A Heuristic Program for Assembly Line Balancing (New Jersey: Prentice-Hall).

Tonge, F. M., 1965, Assembly line balancing using probabilistic combinations of heuristics. M anagement Science, 11, 727-735.

VRAT, P., and VIRAN, A., 1976, A cost model for optimal mix of balanced stochastic assembly line and the modular assembly system for a customer oriented production system. International J ournal of Production Research, 14, 445-463.

Wee, T. S., and Magazine, M. J., 1982, Assembly line balancing as generalized bin packing. Operations Research Letters, 1, $56-58$.

White, W. W., 1961, Comments on a paper by Bowman. Operations Research, 9, 247-277.

Wilhely, W. E., 1987, On the normality of operation times in small-lot assembly systems: a technical note. International J ournal of Production Research, 25, 145-149.

Yano, C. A., and Bolat, A., 1989, Survey, development, and application of algorithms for sequencing paced assembly lines. J ournal of $M$ anufacturing and Operations $M$ anagement, 2, 172-198. 